\title{
Research on Innovation Ability Cultivation and Evaluation Index System of Local High Level Technical Personnel
}

\author{
Chen Lei ${ }^{1, a}$, Gong Ruikun ${ }^{2, b}$, Nian Shanpo ${ }^{3, c}$, Lv Xiuling ${ }^{1, d}$, Ge Fengli ${ }^{4, a,{ }^{*}}$ and \\ Gong Yuhan ${ }^{2, b}$ \\ ${ }^{1}$ Graduate School of Hebei United University,Lunan,Tangshan,Hebei,China \\ ${ }^{2}$ College of Electrical Engineering of Hebei United University,Lunan,Tangshan,Hebei,China \\ ${ }^{3}$ State Owned Assets Department of Hebei United University,Lunan,Tangshan,Hebei,China \\ ${ }^{4}$ College of Management of Hebei United University,Lubei,Tangshan,Hebei,China \\ aclei810@163.com, bgrk@heuu.edu.cn, ${ }^{\mathrm{c}}$ shanpo1999@126.com, ${ }^{\mathrm{d}}$ yjsb@heuu.edu.cn
}

${ }^{*}$ Corresponding author

\begin{abstract}
How to evaluate innovation ability objectively and scientifically of high level innovative technical personnel is of great significance for an area to draw up scientific and technical policy and originality innovation strategy. By literature review method, analytic hierarchy process and expert scoring method, throuh the basis on analyzing a variety of factors which impact on innovation ability of local high level technical personnel, after two rounds of expert consultation, three level evaluation index system has been established, which includes sixteen two-level indexes and thirty five three-level indexes. The corresponding weighting coefficients and the main observation point are provided by every index. For example postgraduates graduating in 2013 from an university in Hebei province, output and effect evaluation of index system are investigated from performance appraisal, which proved that this index system is scientific and feasible.
\end{abstract}

Keywords: High level, Innovation ability, Evaluation index system, Postgraduate.

\section{地方高层次人才创新能力评价指标体系研究}

陈䂞 $^{1, a}$, 龚瑞昆 ${ }^{2, b}$, 粘山坡 ${ }^{3, c}$, 吕秀玲 ${ }^{1, d}$, 葛风丽 ${ }^{4,} a^{*}$, 龚雨含 ${ }^{2, b}$

${ }^{1}$ 河北联合大学研究生学院, 路南, 唐山, 河北, 中国

2河北联合大学电气工程学院, 路南, 唐山, 河北, 中国

3河北联合大学国有资产处，路南，唐山，河北，中国

4河北联合大学管理学院, 路北, 唐山, 河北, 中国

aclei810@163.com, bgrk@heuu.edu.cn, cshanpo1999@126.com, ${ }^{d}$ yjsb@heuu.edu.cn

通讯作者

中文摘要. 通过文献复习法、层次分析法和 专家咨询打分法, 在分析影响地方高层次技
术人才创新能力多种因素的基础上,通过两 轮专家咨询, 建立了三层次评价指标体系, 
该评价体系包括基础实力、师资力量、实践 能力、教师指导、服务设施和绩效评价 6 项 一级指标, 下设 16 项二级指标和 35 项三级指 标, 对每一指标给出相应的权重系数和主要 观测点。以河北省某高校2013届硕士毕业研 究生为例, 从绩效评价方面来探讨指标体系 的产出和效果, 证明本指标体系具有一定的 科学性和可行性。

\section{关键词: 高层次; 创新能力; 评价指标体系;}

\section{研究生}

\section{1. 引言}

经济的日益全球化和高新技术的快速 发展对区域经济的可持续发展提出了新的 更高要求。地方高层次技术人才的创新能力 和素质水平的高低已成为一个地区、乃至整 个国家经济、政治、文化发展的基本决定因 素之一。创新能力是民族进步的灵魂、经济 竞争的核心, 当今社会的竞争是人的创新能 力的竞争。在现阶段经济结构调整和转型的 进程中, 人才的地位和作用更加凸显, 人才 工作已成为重中之重。大量事实证明, 一个 高端人才可以引领和带动一个领域或产业 的发展, 一个领军人物, 可以把一个企业变 成一个品牌。人才是最活跃的生产要素, 决 定着社会生产的广度和深度, 高层次人才是 整个人才队伍建设的龙头和关键。

相比中央和部属高校, 地方高校在技术 人才培养、服务地方经济建设和发展的职能 和职责更为突出和明显。在教育部2013年度 教育统计数据中 ${ }^{[1]}, 2013$ 年地方院校毕业研 究生 229646 人, 占全部毕业研究生 476019 人的 $48.2499 \%$, 在读研究生 789165 人, 占 全部在读研究生 1719818 人的 $45.89 \%$, 其分 布情况见图1。地方高校研究生教育所培育 的广大专业技术人员是各个行业的基层力 量, 为地方经济社会的发展和科技进步发挥 了巨大作用, 做出了突出贡献。引入并不断 强化绩效评价的观念, 可以更好的体现区域 特色, 直接服务于地方的经济和社会发展。

中山大学的黄晓婷 ${ }^{[2]}$ 采用问卷调查法 和层级分析程序法, 分别构建了地方高校科 学学位和专业学位硕士研究生教育的三级 指标体系, 包括条件层面、过程层面、成果
层面 3 个一级指标、 8 个二级指标和 29 个三级 指标。四川大学的王琳等 ${ }^{[3]}$ 以研究型大学为 例, 设计了研究生拔尖创新人才培养绩效评 价体系，含培养甄别、培养过程和培养成果 3 个一级指标、 22 个二级指标及权重。苏州 大学的陈颖 ${ }^{[4]}$ 运用多级模糊综合评价法构 建了涵盖输入指标、过程指标、输出指标在 内的 3 个一级指标、 11 个二级指标和 41 个三 个指标的高等学校硕士研究生培养绩效评 价指标体系。南京航空航天大学的沈春光 ${ }^{[5]}$ 等运用灰色系统理论探讨了区域科技人才 创新能力的灰色综合动态评价方法, 建立了 多层次评价指标体系。因而, 探讨地方院校 研究生教育的学科专业设置与地方经济发 展的关系, 对地方的创新人才培养、经济建 设和科技进步有重要意义。

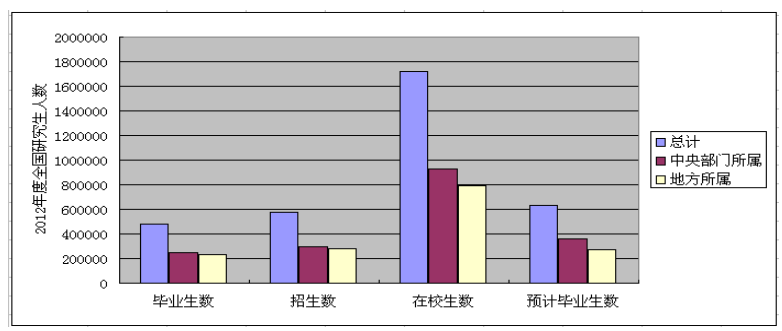

图1 全国研究生2012年度人数类别分布图

\section{2. 概念界定}

为更好的阐述各要素之间的关系, 先对 各关键词的内涵进行说明和界定。

高层次: 指主要从事科研、教学、工程 技术、金融、管理等工作，为国内所急需的 学术技术带头人、高级专业技术人才、高级 管理人才, 或者拥有较好的产业化发展前景 的专利、发明或专有技术，已经取得显著成 绩的专业技术, 符合社会需求的人才, 最好 是专业的工程师和具有硕士学位以上的人 才。文中的高层次指在高校攻读硕士学位的 全日制研究生和在职人员, 即职业前景为较 开阔的高水平专业类工程师和管理人员。

技术人才: 指通过学习、接受某方面或 某领域的技术知识, 具备该专业或该领域的 相应技术能力的人员称为专业技术人员。其 中, 较为突出的熟悉相关技术、具有自主创 新能力的人员, 称为专业技术人才。技术人 才需要借助自身以外的载体来完成任务, 
即, 需要掌握一定的专业工具适用能力。技 术型人才是指掌握和应用技术手段为社会 谋取直接利益的人才, 因而, 他们常处于工 作现场或生产一线工作，常指工程技术人 员、农业技术人员、科研人员、教学人员、 民用航空飞行技术人员、船舶技术人员、经 济人员、会计人员等。文中的技术人才主要 指侧重于工科背景的理工科类研究生。

创新能力也称为创新力。1919年, 我国 著名教育家陶行知先生第一次把 “创造”引 入教育领域。他在《第一流教育家》一文中 提出要培养具有 “创造精神” 和 “开辟精神” 的人才, 阐述培养学生的创新能力对国家富 强和民族兴亡有重要意义。1998年11月24 日, 江泽民同志在新西伯利亚科学城会见科 技界人士时曾指出: “创新是一个民族进步 的灵魂, 是一个国家兴旺发达的不竭动力。 创新的关键在人才, 人才的成长靠教育。”

创新能力 ${ }^{[6]}$ 是指运用知识和理论, 在科 学、艺术、技术和各种实践活动领域中不断 提供具有经济价值、社会价值、生态价值的 新思想、新理论、新方法和新发明的能力。 创新能力按主体分, 最常提及的有国家创新 能力、区域创新能力、企业创新能力等, 并 且存在多个衡量创新能力的创新指数的排 名。文中指可以进行自主学习知识, 融会贯 通, 运用知识解决相应工作中遇到的技术问 题的能力和素质。

2012年6月, 教育部颁布《国家教育事 业发展第十二个五年规划》, 指出, “以学 科为基础, 以改革为重点, 以创新能力提升 为突破口, 建立一批相对独立、集人才培养 和解决重大问题为一体的协同创新平台, 构 建多元、融合、动态、持续的协同创新模式 与机制, 推动知识创新、技术创新、区域创 新的战略融合, 培养一批拔尖创新人才”, “到2015年基本建立科学的质量评价体系 和有效的质量保障体系, 青少年学生身心健 康水平进一步提高, 学习能力、实践能力、 创新能力显著增强。”

\section{3. 创新能力培育的方法和途径}

综观近十几年对创新能力的研究成果, 虽然国内学者的理解各不相同, 但他们对创 新能力内涵的阐述基本上有以下几种观点。

北京电子科技职业学院的安江英等 ${ }^{[7]}$ 认为创新能力不仅表现为对已有知识的获 取、改组和运用, 对新思想、新技术、新产 品的研究与发明, 而且也表现为一种追求创 新的意识, 一种发现问题并积极探求的心理 取向，一种善于把握机会的敏锐性，一种积 极改变自己并改变环境的应变能力。中国矿 业大学的庄寿强等 ${ }^{[8]}$ 认为从创新能力应具 备的知识结构着手, 认为创新能力应具备的 知识结构包括基础知识、专业知识、工具性 知识或方法论知识以及综合性知识四类。

上述三种观点, 尽管表述方法有所不 同, 但基本上能将创新能力的内涵解释清 楚。创新的首要任务之一是提高人们的创造 能力。行为创造学的研究结果给出了个人创

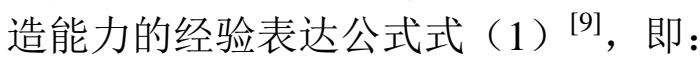

创造能力 $=\mathrm{K}^{*}$ 创造性 $*$ 知识量

式中: $K$ : 为一常量;

创造性：指创造意识、创造人格、创造 思维、创造原理、创造方法之和;

知识量: 指一个人所拥有的知识广度和 相应的深度。

从式(1)可知, 要提高一个人的创造能 力有两个途径可遵循: 一是常见的传统途 径, 即在创造性不变的前提下增加其知识量 (有效增加一个人的知识量是传统教育的 一个优势, 但传统教育在增加人们知识量的 同时, 常常又消弱了其创造性, 所以在传统 教育下, 人的创造能力并不容易获得提高); 二是在一个人现有知识量不变的情况下, 通 过提高创造性的途径来提高其创造能力。大 量的事实已经表明, 后者是提高创造能力的 一条极有效的途径。

鉴于创新能力的多种内涵表述, 可从以 下几个方面对创新能力进行有意识的培育。

\section{1 从个体发展的主观能动性上准确入手}

创新能力是创造能力的某些部分。创造 性是创造能力的一个组成部分, 是创造能力 中除了知识以外的部分。所以, 培养人的创 新能力可以从创造性的五个内涵来进行, 
即: 牢固树立创新意识、全面培养创新人格、 持续训练创新思维、努力掌握创新原理和及 时了解创新方法。通过不断的全面提高个体 的创新性来提高其创新能力, 从而使个体获 取很多的创造或创新成果。

\section{2 从团队协作的集体优越性上持续发力。}

现代社会, 二十一世纪已进入知识经济 的时代, 个体之间的依存关系更为密切, 信 息和知识资源只有通过人们之间的合作才 能得到创新和发展, 人们之间也只有通过相 互的友好合作和交流才能更快更多地获取 知识和信息。所以, 在现代社会的工作和交 往中, 对团队之间相互协作的快捷性、有效 性提出了更高的要求, 只有在良好的团队协 作精神的指引下, 实现社会各领域科学发展 的同时, 达到个体创新能力的提高。

\section{3 从环境氛围的广泛影响性上积极构建。}

创新型国家的建立离不开国民创新能 力的提高, 个体创新能力的提高离不开其创 新性的增强。高等教育肩负着培养数以千万 计的高素质专门人才和一大批拔尖创新人 才的重要使命, 提高研究生的培养质量, 是 高等教育自身发展规律的需要, 也是办好让 人民满意的高等教育的需要。提高研究生的 创新能力和创业能力, 更是建设创新型国 家、构建和谐社会主义社会的需要。必须进 一步推动创新能力培育氛围的宣传建设, 鼓 励和支持研究生尽早参与科学研究、技术开 发和社会实践等创新活动, 提高他们的科学 素质、文化素养、创业精神和实践能力。

\section{4. 创新能力的评价指标体系}

新形势下, 为使地方高校攻读硕士研究 生人员在迅速变化的环境中具有生存、立足 和发展的基础, 具有良好的解决实际问题的 综合技术能力, 必须加强其创新精神和创新 能力的培养, 在构建研究生创新能力的评价 指标体系时, 应遵循以下的一些具体原则。

\section{1 全面性原则}

确保评价指标体系具有全面性、重点 性、先导性。评价指标体系应能对理工科研 究生的创新能力的培育有明确的指导作用。 因此, 既要充分反映地方院校研究生质量现
状和开展创新能力的条件、途径的实际, 还 要对研究生教育的科学发展有指导作用。

\section{2 整体性原则}

对研究生创新能力培育指标体系的设 计必须客观、全面、有侧重的选取与培养对 象有作用或影响的指标, 做到公正公平。因 评价体系涉及到的指标因素有很多, 对于指 标体系的设计, 必须从地方院校到研究生和 用人单位全面、有主次性地考虑这些指标。

\section{3 科学性原则}

科学性原则主要体现在理论和实践相 结合, 在理论上要有依据, 以及所采用方法 具科学性等方面。抓住最重要的、最本质的 和最有代表性的东西, 建立能客观描述研究 生创新能力的抽象模型, 使评价指标体系能 够在逻辑结构上与实际结合得更严谨合理。

\section{4 定量、定性相结合的原则}

培育研究生的创新能力相关的评价指 标比较复杂, 设计指标体系时, 主要运用定 量指标进行各个影响因素的分析和评价。采 用定量化的评价指标能够更为明确地反映 出各影响因素对研究生创新能力的作用强 弱。同时，对于某些目前不易定量分析的指 标, 可以采用定性分析、模糊判断等方法。

\section{5. 效果分析}

基于创新能力的几种内涵、培育方法和 评价原则, 结合黄晓婷、王琳等人的研究成 果, 提出基于地方高校的硕士研究生的创新 能力的评价指标, 采用层次分析法和教育专 家打分法, 经过二轮反复研究后, 给出涵盖 主体、客体及效果评价的包含基础实力、师 资力量、实践能力、教师指导、服务设施和 绩效评价的六个一级指标, 下设专业背景、 学习意愿、教师水平、教师教育、学生评价、 课程阶段、论文阶段、课程情况、论文指导、 科研交流、学习场所、学习资源、课程成绩、 论文质量、荣誉称号和发展前景等十六项二 级指标, 又分解为三十五项三级指标的指标 体系, 并赋予各个指标相应的权重系数。

在提出研究生创新能力和绩效评价指 标体系的基础上, 以河北省某高校2013届毕 业硕士研究生为例, 从绩效评价方面来探讨 指标体系的产出和效果情况。 
在2013年度研究生国家奖学金评定结 果的统计中, 有五十三位毕业生获此殊荣, 占可参评全体研究生的 $3.87 \%$ 。在优秀硕士 学位论文的评选中, 七人获 2012 年河北省优 秀硕士学位论文, 占获奖总人数的 $7 \%, 2013$ 春季有二十六篇硕士论文、夏季有三十九篇 硕士论文荣获校级优秀硕士学位论文荣誉 称号, 占全体毕业研究生的 $10.09 \%$, 有七 十人获2013届春季、夏季毕业优秀毕业研究 生, 占 2013 年度毕业总人数的 $10.82 \%$ 。有 四人获省级三好研究生, 共计 198 人次获校 优秀研究生干部、三好研究生等荣誉称号, 占可参评全体研究生的 $9.34 \%$ 。有 16 人成功 考取博士研究生, 继续深造, 其他毕业生都 顺利走上工作岗位, 较好地服务于社会。

综上, 创新能力绩效培育及评价指标体 系基本上能够反映出评价研究生创新能力 的各个方面, 而且具有一定的可操作性。

\section{4. 结束语}

随着高层次高等教育的深入发展和培 育模式的不断创新, 经济社会发展对地方高 层次专业技术人才需求的变化, 创新能力评 价的各级指标和观测点需要进一步的优化 和完善, 本指标体系中的指标设置、权重赋 值等需要做出相应的更具量化标准的动态 调整, 使其更具科学性和可操作性, 以更好 地实现对人才创新能力培育的合理评价。从 而, 进一步发展壮大地方高层次人才队伍, 尽快形成地方高层次人才独特优势, 为当前 经济结构的调整和转型, 较好地实现跨越式 发展提供强大的人才支撑和智力保证。

\section{致谢}

本文为唐山市科技计划项目《唐山市高 层次创新型应用技术人才培育研究》 (12140207b)和河北联合大学研究生教育教 学项目《研究生创新能力及绩效评价体系研
究》(C1101)、在职工程硕士研究生教育质 量评价研究(Z1303) 的阶段性成果之一。

\section{References}

[1] ht.tn://www. moe. gov. cn/nublicfiles /hris iness/htm1 fi les/moe/s7567/201 309/156773. html.

[2] Huang Xiao-Ting. The construction of nerformance evaluation index of nostoraduate education in I ncal colleges and Universities. Journal of Pioneerng with Science and Tehnology Monthly, pp. 88-90, 2008, (5).

[3] Wang Lin.Chang guang-dong and $\mathrm{Wu}$ Yao-hong. Research on nerformance evaluation of postgraduate students top-notch creative talents training. .Journal of Science and Technologv Management Research, pp. 75-78, 2010, (13).

[4] Chen ving. The studv on nerformance evaluation of master students in higher learning institutions. Master Dissertation of Soochow University, 2009, (4).

[5] Shen chun-guang. Chen wan-ming and Pei ling-ling. A research on the evaluatin targets svstem and method of the capailitv of regional technical innovative personal. Science of Scienc and Management, pp. 196-199, 2010, (2).

[6] Zhang bao-chen. Reform of normal education and develonment of innovative abilitv for primarv and middle school students. Journal Theorv and Practice of Education, vol. 24, pp. 40-42, 2004.

[7] An Jiang-ving and Wang Li-ming. Building the model of training high skilled talents. Journal of Chinese Vocational and Technical Education, pp. 67-71,75, 2012, (24).

[8] Zhuang Shou-aiang. An analvsis of innovation.creation and other concents related to higher education. Journal of Meitan Higher Education, vol. 22, pp. 66-71, 2004.

[9] Zhuang Shou-aiang. Relationship among innovational-tvpe state. creativeness and creatologv. The International Conference on the Science of Creation, pp. 20-22, 2006. 